\title{
The Role of Entropy in Design Theory and Methodology
}

\author{
Waseem A. Khan and Jorge Angeles \\ Department of Mechanical Engineering \& Centre for Intelligent Machines \\ McGill University \\ 817 Sherbrooke Street West, Montreal, Quebec, Canada H3A 2K6 \\ angeles@cim.mcgill.ca
}

\begin{abstract}
We outline here the pertinence of the concept of entropy in design theory and methodology. We do this by resorting to the concept of design complexity. The concept is illustrated with one example.
\end{abstract}

\section{Introduction}

The word "design" is derived from the Latin "designare," meaning "to mark out." This word bears many meanings in English, as it is used as a noun and as a verb. As a noun, moreover, the "D" word is used to designate the product, the process, the visual representation, etc.

As a verb, to design is to conceptualize a product, tangible or intangible, intended to satisfy a human need. As a noun, design is a broad concept, including: a) the sublime, e.g., a masterpiece of art like The Mona Lisa; b) the pragmatic, e.g., a mass transport system; c) the intangible, like a piece of code; and d) the concrete, like a bridge joining two pieces of land.

Design is thus a broad activity, exhibiting several manifestations. In ascending order of technical content, we have: art; graphic design; industrial design; architectural design; and engineering design. Sometimes, the last two are referred to as technical design. The term "technical" derives from Greek $\tau \eta \chi \nu \eta$ (techneh), which means art or craft. Technical bears the connotation of material realization. Art itself has its technical content as the painter, the sculptor, the composer must obey certain rules that govern their medium of expression and that are independent of the purely artistic content of their work. However, the artistic, emotive content of art is much higher than that of industrial design, and is superseded, to a certain extent, by technical content in engineering design.
Engineering design is a pervasive activity that appears in every engineering project. Engineering design is also heavily dependent on subjective concepts, such as thinking processes. Although attempts have been made to formalize engineering design as a science, the appearance of various, quite disparate, schools of thought indicates that the theory of engineering design is an intellectual activity in evolution, whereby research is still needed to lay its foundations in a broadly acceptable framework.

This paper is an attempt to lay the foundations of a design theory within the engineering realm. Such a theory should be built on design principles that are common to all engineering activities, regardless of the underlying disciplinary details. A design theory should thus be equally applicable whether the objective of the task at hand is a coffee filter or a low-pass filter, a Wheatstone bridge or Confederation Bridge.

\section{The Nature of Design}

Design at large aims at satisfying a human need. In its most sublime form, art, the human need is intellectual; in its most pragmatic form, engineering design, the need is physical.

In a design job there is always a client, who submits the need to the designer, be this an individual or a design team. It is only seldom that one single individual is capable of undertaking and successfully completing a design job.

As outlined above, design is a complex activity that draws from creativity, scientific and engineering knowledge as well as hard and soft skills (team work, communication, interpersonal relations, and so on). Because of its complexity, design researchers have devised models of the design process. The three most frequently cited models are those due to 


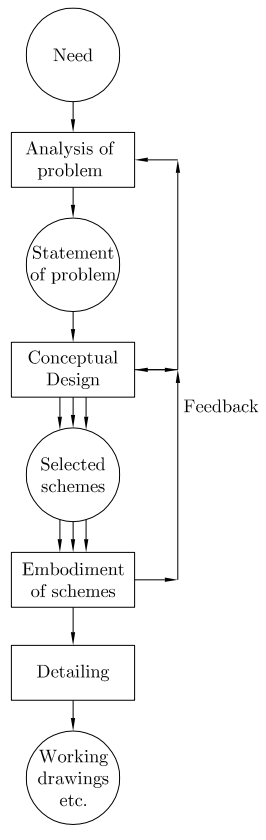

Figure 1: Design process model after French (1999)

French [1], Pahl et al. [2] and the VDI ${ }^{1}$ [3]. Shown in Fig. 1 is a flow diagram of the first model. Block diagrams of the second and third models are available as Figs. 4.3 and 1.9 in [2].

In the foregoing models we can identify three major stages (or steps) in the design process, namely,

1. Conceptual design: first, the need motivating the design job is analyzed by the designer in consultation with the client, and then formulated in terms of functions and subfunctions; more precise conditions are also included, as per the client's needs and resources, these conditions constituting the design specifications commonly referred to as the design specs. Then, a rich set of alternative solutions is generated by means of creativity-enhancing methods (brainstorming, synectics, IPS, etc.); finally, this set is thoroughly analyzed and scrutinized, until a reduced set of alternatives is identified as the candidate solutions that stand the highest chances of meeting all the functions and specifications, while observing all the constraints (timelines, budget, etc.)

2. Embodiment design: the alternatives identified in item 1 are given form by means of

\footnotetext{
${ }^{1}$ This is the acronym of Verein Deutscher Ingenieurer or Association of German Engineers.
}

sketches and preliminary design drawings; several criteria are then put in place in order to decide which of these alternatives is the most likely to satisfy the client. In complex design jobs, like in aircraft design, a parametric model of the selected alternative(s) is(are) produced, with purposes of analysis and, eventually, optimization of the designed object.

3. Detail design: Once the design team has zeroed-in on one design solution, all parts and components are either designed or selected from catalogue. The final product is a design report, containing: a summary of the need that motivated the job; all design solutions considered; a thorough description of the design solution of choice; the manufacturing drawings; tables; plots; estimated budget to produce the object designed; and all kinds of information needed to understand the proposed solution.

It is then apparent that at the outset, at the conceptual stage, a mathematical model is not available. This will come once an embodiment of a solution has been produced. At the conceptual stage, only rules of the if ... then type are possible. These rules are heuristic, in that they are based on common sense and experience, more so than on solid science and engineering knowledge.

Some tools have been developed over the years to help the engineer at the early design stages. These have been proposed in the form of principles that are applicable to all engineering design jobs, regardless of the discipline. Three main schools are to be cited: The German School; Robust Design; and Axiomatic Design.

The German School is highly developed, with guidelines approved and provided to the public by the VDI. Robust design finds its roots in Genichi Taguchi's Robust Engineering. Taguchi's paradigm draws from concepts of communications engineering, as Taguchi is a communications engineer by training. While robust engineering was proposed by Taguchi in the fifties, the paradigm remained virtually unknown outside Japan, until Taguchi's work was translated into English in the early eighties. Axiomatic design was proposed by MIT's Nam P. Suh $[4,5]$. Suh's paradigm is based on two main "axioms," as Suh calls them²:

Axiom 1: The Independence Axiom. Maintain the independence of the functional requirements; and

\footnotetext{
${ }^{2}$ A more appropriate term would be "principles"
} 
Axiom 2: The Information Axiom. Minimize the information content of the design.

Taguchi's paradigm is based on the concepts of robustness, which is measured in terms of the signalto-noise ratio: the higher the ratio, the more robust the design. Suh's paradigm, in turn, is based on the concepts of design matrix and information content, the latter borrowed from the mathematical theory of communication [6]. The concept of information content, in turn, derives from the thermodynamic concept of entropy, as proposed by Rudolf Clausius (1822-1888) in 1865. Clausius introduced the concept in a seminal paper that shed light in the Second Law of Thermodynamics. According to Clausius, "if an isolated thermodynamic system is left alone, entropy can only increase."

\section{Entropy in the Mathematical The- ory of Communication}

The mathematical theory of communication (MTC), sometimes referred to as information theory, or even as cybernetics, is a far more recent discipline than statistical thermodynamics, or than classical thermodynamics, for that matter. The credit of the creation of the theory goes to Claude E. Shannon, who published his seminal paper, "A mathematical theory of communication," in the Bell System Journal in July and October, 1948. In fact, the history of Shannon's theory has been traced back [6] to Boltzmann (ca. 1894), then to Szilard [7], von Neumann (ca. 1932) and Wiener [8], one of the creators of the discipline of cybernetics.

The main issue in MTC is information, and how to quantify the amount of information in a message, which is important in order to measure the capacity, in bits/s, of a communication channel. Within the MTC the concept of information is different from its connotation in everyday's language. As Weaver put it, "information in communication theory is associated with the amount of freedom of choice we have in constructing a message" [6]. In this context, freedom is to be understood as the opposite of order: A perfectly ordered society is one overwhelmed with laws and rules, to the point that it removes freedom from its individuals. A society without laws and rules, on the contrary, has complete freedom, but becomes totally disordered.

Shannon's notion of information, or its content in a message, in fact, can also be understood as the level of ambiguity of a message. Hence, contrary to the notion of information in everyday's language, information in the MTC is something we want to keep to a minimum, a perfect message carrying zero ambiguity and hence, zero information.

\section{Design Complexity}

In formulating the Independence Axiom, Suh first defines the functional requirements and the design parameters, to be determined by the designer. In the next step, Suh presupposes linear relations between the two foregoing items, related by a design matrix. Within the paradigm of axiomatic design, the design matrix would better be square, although rectangular matrices can also be accommodated. Indeed, Suh calls "ideal" a design job in which the number of design parameters equals that of functional requirements, thus leading to a square design matrix; a "coupled design" is one design task in which the number of design parameters is smaller than that of functional requirements; the opposite of a coupled design, in turn, is called "redundant." Apparently, in the last two instances the design matrix is rectangular. However, this framework faces some problems when trying to apply it to a concrete design task. The first problem is that, at the conceptual stage, there is no mathematical model available, not to speak of a linear model, to begin with. The second problem arises when attempting to compute the information content of a design. Suh resorts to the concept of entropy in the mathematical theory of communication, as proposed by Shannon [6]):

$$
H=-\sum_{1}^{n} p_{i} \log \left(p_{i}\right), \quad \sum_{1}^{n} p_{i}=1
$$

where $\log \left(p_{i}\right)$ is the $\operatorname{logarithm}$ of $p_{i}$ to a certain base. If we make abstraction of the Boltzmann constant in the definition of the thermodynamic entropy [9] and use natural logarithms in eq.(1), we find that the entropy expressions in the two cases are identical. While in thermodynamics the base $e$ of natural logarithms is preferred, in information theory we are free to choose the logarithm base. The natural choice is binary logarithms, namely, those that use 2 as a base, in which case $H$ is measured in bits. If the Neperian logarithms are used, those to a base $e$, then the unit of measure is the nat; if decimal or Briggs logarithms are used to measure $H$, then the units are decibels.

In information theory, the base 2 is preferred mainly because the theory is based on the concept of choice, which can always be reduced to a binary 


\begin{tabular}{||c|r||}
\hline alpha & November \\
\hline bravo & Oscar \\
\hline Charlie & papa \\
\hline delta & Quebec \\
\hline echo & Romeo \\
\hline foxtrot & sierra \\
\hline golf & tango \\
\hline hotel & uniform \\
\hline India & Victor \\
\hline Juliett & whiskey \\
\hline kilo & xray \\
\hline Lima & yankee \\
\hline Mike & zulu \\
\hline
\end{tabular}

Table 1: The International Alphabet

search. For example, to choose one letter of the 26 of the English alphabet, we can always start by deciding between two halves, the first comprising letters $A$ to $M$, the second from $N$ to $Z$. Then, we would continue with a splitting of each half into two halves, which needn't be of the same size, and so on.

Some examples will clarify the above concepts. Germans have invented tricks to avoid ambiguities when communicating either by telephone or by handwritten messages. For example, when pronouncing zwei (two in English), which rhymes with drei (three in English), on the telephone, they avoid ambiguity by saying rather zwo, which doesn't rhyme with any other digit in German. Likewise, when handwriting a word that is not common, e.g., a proper name, which contains a combination of u's, n's, w's and m's, to avoid confusing u with n, they overline the u's; ditto the w's.

As a matter of fact, the International Civil Aviation Organization (ICAO) devised its International Alphabet with the aim of avoiding ambiguities when communicating via a noisy aviation channel. Notice that the noise of the channel is exacerbated by the noise generated by the speaker herself or himself, when speaking a foreign language, or when speaking while suffering of a cold. Shown in Table 1 is the International Alphabet.

Let us assume that we want to spell a name by telephone: Anne. We could choose to spell each letter as such, in which case we would risk the ambiguity of homophones, as

$A$ can be understood as $A$ (rightly), but also as eight or $H$, which gives us a probability of $1 / 3$ for being correctly understood for the first letter;

$N$ can be understood as $N$ (rightly), but also as $M$, thus giving us a probability of $1 / 2$ for being correctly understood.

$E$ can be understood as $E$ (rightly), but also as $B, C, D, G, P, T, V$, which gives us a probability of $1 / 8$ for being correctly understood.

Thus, we have the information content of each of the four messages, one for each letter:

$$
\begin{aligned}
H_{A} & =-\sum_{1}^{3} \frac{1}{3} \log _{2}(3) \\
& =3 \times \frac{1}{3} \times 1.5850=1.5850 \\
H_{N} & =-\sum_{1}^{2} \frac{1}{2} \log _{2}(2) \\
& =2 \times \frac{1}{2} \times 1=1.0 \\
H_{E} & =-\sum_{1}^{8} \frac{1}{8} \log _{2}(8) \\
& =8 \times \frac{1}{8} \times 3=3.0
\end{aligned}
$$

Hence, the total information content of the four messages becomes

$$
\begin{aligned}
H & =H_{A}+2 \times H_{N}+H_{E} \\
& =1.5850+2 \times 1.0+3.0=6.5850 \mathrm{bits}
\end{aligned}
$$

On the other hand, if we choose to use the words of the International Alphabet, then, we remove the ambiguity, which leads to $H_{A}=H_{N}=H_{E}=0$, the total information content thus reducing to zero.

Suh proposes to interpret the $p_{i}$ in eq.(1) as the probability of meeting a tolerance. However, at the conceptual stage, we do not have as yet a parametric model of the object under design, and hence, we cannot associate a tolerance to each parameter.

What we propose here is to regard the entropy of a message or of a design concept produced, e.g., during a brainstorming session, as the complexity of this concept. Here, we liken complexity to diversity: The more diverse the design concept at hand, the more complex it is. In other words, the higher the diversity content of an alternative solution to a design problem, the more complex the solution is.

In order to assess the diversity of a design alternative, we will introduce first some definitions: 
Function: A generic task imposed by the need to be satisfied by means of the object under design. Examples: fasten; separate; sort; support; transport; energize; entertain; actuate; sense; etc.

Function-carrier: A component or assembly intended to implement a function [2]. Examples: a bolt, a welding seam or a screw are components that serve the function fasten, and hence, they are carriers of this function.

Design specification: A quantitative condition to be met by the object under design. Examples: A system to transport persons and materials through a span of $0.5 \mathrm{~km}$ should serve 1000 people/h and transport 1000 ton/h of merchandise.

Functions have subfunctions. Example: The function separate can have the subfunction allow relative rotation of two neighbouring disks about one common axis.

Let us assume that a given function $F$ entails $N$ subfunctions $f_{1}, f_{2}, \ldots f_{N}$, with $f_{i}$ to be implemented with a number $\nu_{i}$ of identical carriers $C_{i}$, so that the total number $N_{c}$ of function-carriers for $F$ is

$$
N_{c}=\nu_{1}+\nu_{2}+\ldots+\nu_{N}
$$

Let us now denote with $\phi_{i}$ de frequency of occurrence of function-carrier $C_{i}$, namely,

$$
\phi_{i}=\frac{\nu_{i}}{N_{c}}, \quad i=1, \ldots, N
$$

and hence,

$$
\sum_{1}^{N} \phi_{i}=1
$$

Apparently, the frequencies $\phi_{i}$ in eqs.(6) \& (7) play the role of the probabilities $p_{i}$ in eq.(1). We can thus define a diversity in the design-solution alternative at hand as the equivalent of the entropy in eq.(1). It would just be fair to refer to this design entropy as the design complexity $K$, namely,

$$
K=-\sum_{1}^{N} \phi_{i} \log _{2}\left(\phi_{i}\right)
$$

It is noteworthy that Shannon's formula, eq.(1), is an approximation to a combinatoric number, as appearing in statistical thermodynamics [9]. This approximation, based on the Stirling formula, is quite accurate for "large" values of $N$, as the numbers of particles occurring in thermodynamic systems are huge. The numbers $N_{c}$ of function carriers in a design are comparatively modest. Nevertheless, the Stirling formula gives a relatively low error, of about $14 \%$, for numbers of the order of 10 [9], and hence, can be adopted within a design-theoretical framework.

As a matter of example, let us consider a concrete design problem:

Design an autonomous vehicle capable of transporting bundles of veneer in a production plant.

After a brainstorming session, the design team came up with several alternatives for a design solution, the preferred choice being a tricycle, provided with three conventional wheels ${ }^{3}$ of identical size and shape (minimum diversity of wheel models!). Moreover, the vehicle, to be autonomous, will be programmable either offline or online, which means that its motors will be under computer control. A vehicle of this kind is constrained to move in a direction dictated by the orientation of its wheels. Furthermore, this means that the vehicle has a mobility of 2 , as each wheel can only move under driving and steering.

From the above discussion, it is apparent that the vehicle will need two motors, the question being, still at the conceptual design stage, where to place the two motors, as one has three wheels at her or his disposal. Furthermore, we have to consider that in a tricycle, two wheels are mounted on coaxial shafts, with their axes fixed to the vehicle body. This means that these two wheels can only be actuated under driving, but not under steering. The third wheel can be both driven and steered. Additionally, this wheel can be either centred or offset. We thus have a few alternatives, as depicted in Fig. 2.

Apparently, the choice of actuation mode boils down to using either two distinct motors, each with its own control hardware and software, or two identical motors under the same control hardware and software. Obviously, the second alternative is more promising, as it entails the lower diversity, and hence, the lower complexity, namely, 0 . The complexity of the first alternative is 1.0 , as the reader can readily verify.

\footnotetext{
${ }^{3}$ In mobile robotics nonconventional wheels are those with morphologies more complex than conventional wheels consisting essentially of one disk; one type of these is Mekanum wheels, which consist of a drum with a series of rollers on its periphery, at an angle with the axis of the drum, which provides full mobility to the wheel, i.e., the ability to move sideways while rolling.
} 


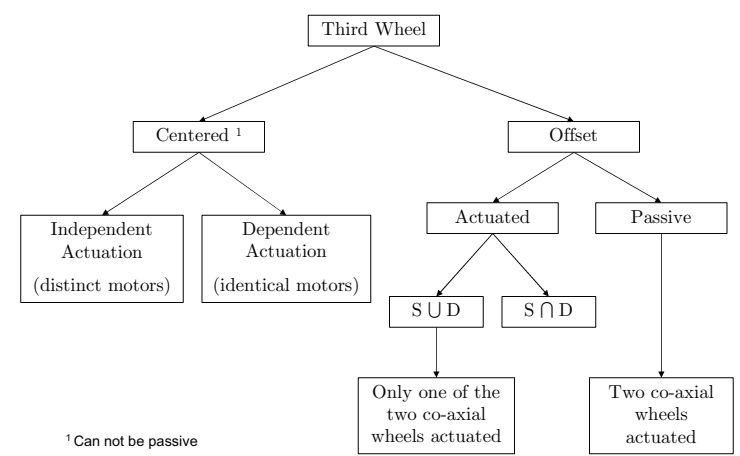

$\mathrm{S}=$ Steered

$\mathrm{D}=$ Driven

Figure 2: Actuation alternatives for an autonomous tricycle

Once we have zeroed-in on the use of two identical motors, the question is how to implement each of the two. This is the subject of embodiment design, the second stage in the design process. We will not elaborate on this issue here.

\section{Conclusions}

We proposed complexity as a measure of the diversity content of a design solution at the conceptual stage. The complexity measure proposed here is computable with the information content encountered in the mathematical theory of communication and entropy in statistical thermodynamics.

\section{Acknowledgements}

The support of the NSERC Design Engineering Chair "Design for Extreme Environments" and of McGill University through a James McGill Professorship to the second author is duly acknowledged.

\section{References}

[1] French, M., Form, Structure and Mechanism, Springer-Verlag, London, 1992.

[2] Pahl, G., W. Beitz, J. Feldhusen, and K.H. Grote, Engineering Design: A Systematic Approach, Third Edition, Wallace, K.M. and Blessing, L. (translators and editors), SpringerVerlag London Ltd., London, 2007.
[3] VDI, Richtlinie 2221: Methodik zum Entwickeln und Konstruieren technischer Systeme und Produkte (Systematic Approach to the Design of Technical Systems and Products), VDI-Verlag, Düsseldorf, 1993.

[4] Suh, N.P., The Principles of Design, Oxford University Press, New York, 1990.

[5] Suh, N.P., Axiomatic Design. Advances and Applications, Oxford University Press, Oxford, 2001.

[6] Shannon, C.E. and W. Weaver, The Mathematical Theory of Communication (first published in 1949), University of Illinois Press, Urbana and Chicago, 1998.

[7] Szilard, L., "Über die Entropieverminderung in einem thermodynamischen System beim Eingriffen intelligenter Wesen" (On the reduction of entropy in a thermodynamic system by the intervention of an intelligent being), Zeitschrift für Physik, 1929, Vol. 53, pp. 840-856.

[8] Wiener, N., Cybernetics, The Technology Press of M.I.T. and John Wiley \& Sons, Inc., New York, 1948.

[9] McQuarrie, D.A. and J.D. Simon, Molecular Thermodynamics, University Science Books, Sausalito, CA, 1999. 